\title{
VERY HIGH RESOLUTION OBSERVATIONS OF THE LUMINOUS WATER MASERS IN NGC 4258
}

\author{
M. J. Claussen (FCRAO, Univ. of Massachusetts, Amherst, MA USA), \\ M. J. Reid, M. H. Schneps (CfA, Harvard Univ., Cambridge, MA USA), \\ K.-Y. Lo (Univ. Illinois, Urbana, IL USA), \\ J. M. Moran (CfA, Harvard Univ., Cambridge, MA USA), and \\ R. Gusten (MPI, Bonn, FRG)
}

\begin{abstract}
We summarize the results and interpretation of a four station transcontinental VLBI experiment of the luminous water masers in the nearby galaxy NGC 4258. At a distance of 5 $\mathrm{Mpc}$, the longest baseline of the experiment provides spatial resolution of less than $10^{16}$ $\mathrm{cm}$. The strongest maser emission was detected on all baselines, and was found to consist of at least two features separated by about 0.1 mas $\left(10^{16} \mathrm{~cm}\right)$. Weaker features are possibly spread over a region up to 1 mas in size. These results provide evidence that supports the scenario described by Claussen and Lo (1986) which suggests that the very luminous water masers reside in molecular gas that immediately surrounds the central, active nucleus.
\end{abstract}

\section{Introduction}

Five nearby galaxies have been found to have very luminous water masers residing in or near the nucleus (see Claussen and Lo 1986 and references cited therein). The total isotropic luminosity of the masers in these galactic nuclei range from 30 to $500 \mathrm{~L}_{\mathrm{O}}$. High resolution observations (35 mas) using the Very Large Array of the NRAO have shown that, for NGC 1068, NGC 4258, and NGC 3079, all the masers are coincident and in the nucleus of their respective galaxy (Claussen and Lo 1986, Haschick et al. 1987). When compared with the size scales and luminosities of masers in the Galaxy, these values are quite remarkable in that a large number of maser sites are required in a rather small volume. It is noteworthy that all five galaxies show some evidence for nuclear activity.

Three competing suggestions have been made in order to explain the origin of such luminous masers: 1) the masers are associated with the active nucleus of each galaxy and may reside in a circumnuclear disk of dust and molecular gas (Lo and Claussen 1986); 2) the masers arise from foreground gas which amplifies background continuum radiation (Haschick and Baan 1985); or 3) the masers are the very high end of a luminosity function of masers similar to those found toward star forming regions in the Galaxy (Ho et al. 1987). In order to determine the spatial distribution of the masers in the nucleus, and to help to distinguish these suggestions, very high resolution observations are necessary. 


\section{Observations, Analysis and Results}

A VLBI spectral line experiment of the masers in NGC 4258 was performed on October 7, 1984, using a transcontinenal array with the following stations: the 40-m at OVRO, the phased VLA, the 43-m at NRAO-Green Bank, and the 100-m at Effelsberg, FRG. The longest baseline (OVRO-Eff) provides a fringe spacing at $22 \mathrm{GHz}$ of about 0.2 mas. The Mark II recording system was used, providing an instantaneous bandwidth of $2 \mathrm{MHz}$ (27 $\mathrm{km} \mathrm{sec}{ }^{-1}$ ). Since this velocity coverage is insufficient to observe all the emission from NGC 4258, switching between video converters set at different center frequencies was used to cover $6 \mathrm{MHz}$. The switching was done at a rate of $1 \mathrm{~Hz}$. The data were correlated at the NRAO spectral line processor in Charlotesville, VA, and standard software was used to perform necessary calibration. The data has been analyzed using both fringerate and fringe-phase techniques. The results of fringe-phase analysis from the longest baseline shows that the strongest feature in the spectrum is split into two components separated by about 0.1 mas, as shown in Figure 1. Fringe-rate analysis on weaker features in the spectrum show that they lie within 1 mas of the strong feature.

These results, taken in conjunction with similar results on NGC 3079 (Haschick et al. 1987), show that the maser emission in these nuclei is distributed in regions of circum-

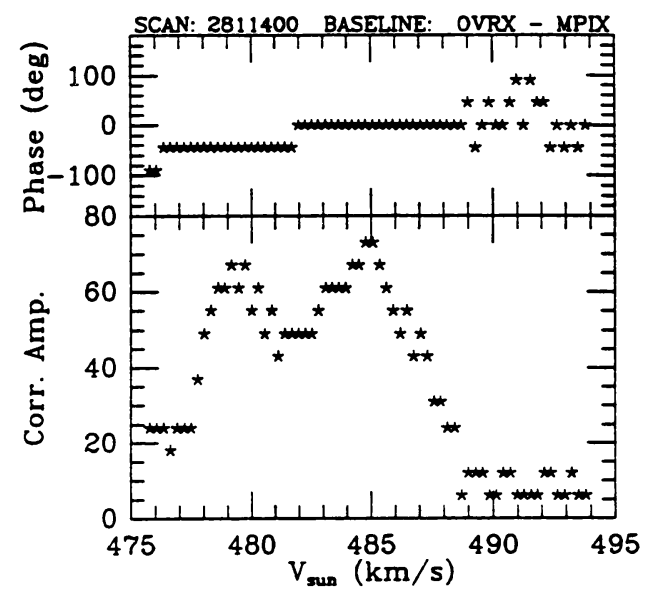

Figure 1 Cross-correlation spectrum of strong water maser feature in NGC 4258 stellar rather than interstellar sizes. It seems unlikely that a single star-forming region (typical Galactic size of $\sim 10^{19}$ $\mathrm{cm}$ ) could give rise to such luminous masers. If the luminous masers are simply the high-luminosity tail of a smooth distribution then one might expect to see more galaxies with nuclear masers of a few $L_{0}$. Present survey statistics do not support such a hypothesis (see Claussen and Lo 1986, Haschick and Baan 1985, and Henkel et al. 1985). Systematic velocity/spatial offsets could determine the kinematic structure of the gas in this region. Aperture synthesis techniques on this data set are now being used in order to determine if such structures exist.

\section{References}

1. Claussen, M. J. and Lo, K.-Y. 1986, Ap. J, 308, 592.

2. Haschick, A. D., Schneps, M. H., Reid, M. J., Baan, W. A., and Moran, J. M., 1987, this volume.

3. Haschick, A. D. and Baan, W. A. 1985, Nature. 314, 144.

4. Henkel, C., Gusten, R. Downes, D., Thum, C., Wilson, T. L., and Biermann, P. 1984, Astr. Ap., 141, L1.

5. Ho, P. T. P., Martin, R. N., Henkel, C. and Tumer, J. L. 1987, preprint. 